Rapid finite-frequency

microseismic noise source

inversion at regional to global

scales

\author{
Journal Article \\ Author(s): \\ Igel, Jonas K.H.; Ermert, Laura A.; Fichtner, Andreas \\ Publication date: \\ 2021-10 \\ Permanent link: \\ https://doi.org/10.3929/ethz-b-000488185
}

Rights / license:

Creative Commons Attribution 4.0 International

Originally published in:

Geophysical Journal International 227(1), https://doi.org/10.1093/gji/ggab210 


\title{
Rapid finite-frequency microseismic noise source inversion at regional to global scales
}

\author{
Jonas K.H. Igel ${ }^{1}$, Laura A. Ermert ${ }^{2}$, Andreas Fichtner ${ }^{1}$ \\ ${ }^{1}$ Institute of Geophysics, ETH Zurich, Switzerland.E-mail: mailto:jonas.igel@erdw.ethz.chjonas.igel@erdw.ethz.ch \\ ${ }^{2}$ Department of Earth and Planetary Sciences, Harvard University, US
}

\section{SUMMARY}

Ambient noise cross-correlations can be used as self-consistent observables, opening novel possibilities for investigating ambient noise sources. To optimise the forwardmodelling of global ambient noise cross-correlations for any given distribution of noise sources in the microseismic frequency range up to $0.2 \mathrm{~Hz}$, we implement (i) pre-computed wavefields and (ii) spatially variable grids. This enables rapid inversions for microseismic noise sources based on finite-frequency source sensitivity kernels. We use this advancement to perform regional and global gradient-based iterative inversions of the logarithmic energy ratio in the causal and acausal branches of micro-seismic noise cross-correlations. Synthetic inversions show promising results, with good recovery of the main dominant noise sources of the target model. Data inversions for several consecutive days at the beginning of October 2019 demonstrate the capability of inverting for the spatio-temporal variations of the sources of secondary microseisms in the ocean. This paves the way for daily ambient noise source inversions which could help improve full-waveform ambient noise tomography and subsurface monitoring methods.

Key words: seismic noise, computational seismology, numerical modelling, waveform inversion 


\section{INTRODUCTION}

Studying the sources of the omnipresent ambient noise field is valuable for our understanding of atmosphere - ocean - solid Earth coupling. The source physics of seismic ambient noise have been investigated intensively and are considered well-understood (Longuet-Higgins 1950; Hasselmann 1963; Ardhuin et al. 2011; Ardhuin \& Herbers 2013; Ardhuin et al. 2015, 2019). Seismic noise between frequencies of around $0.05-0.2 \mathrm{~Hz}$ can be classified into primary and secondary microseisms. Primary microseismic sources with a frequency between 0.05 and $0.1 \mathrm{~Hz}$ are predominantly excited along coastlines by the interaction of ocean waves with the sloping ocean floor. Secondary microseisms, in contrast, originate from interfering ocean waves and are predominantly excited in the open ocean between frequencies of 0.1 and $0.2 \mathrm{~Hz}$.

Recent developments in Distributed Acoustic Sensing (DAS) enabled the direct observation of primary and secondary microseism sources at the ocean floor, thereby directly confirming their source mechanisms (Sladen et al. 2019; Williams et al. 2019). In addition to oceanographic observables such as wave height, seismic noise cross-correlations may contribute constraints on the current and past ocean state alongside pre-existing theories.

While seismic noise sources are interesting in their own right, they also play a major role in ambient noise interferometry. Since the first successful applications of the method (e.g. Aki 1957; Shapiro \& Campillo 2004; Shapiro et al. 2005; Sabra et al. 2005), it has risen in popularity to obtain knowledge of the subsurface without the need for earthquakes or active sources. Most noise tomography methods are based on the assumptions that the noise source distribution is quasi-random over sufficiently long time intervals, and that the wavefield is equipartitioned (Nakata et al. 2019). If these assumptions are made, the cross-correlation converges towards the inter-station Green's function (Lobkis \& Weaver 2001; Wapenaar 2004; Weaver \& Lobkis 2004, Weaver 2008; Shapiro \& Campillo 2004; Wapenaar \& Fokkema 2006; Sánchez-Sesma \& Campillo 2006; Fichtner \& Tsai 2019) which permits the application of established tomography methods, originally developed for use with deterministic point sources. However, the noise source distribution on Earth has strong spatio-temporal variations which potentially corrupt méthods based on Green's function retrieval (Stehly et al. 2008; Halliday \& Curtis 2008; Tsai 2009; Kimman \& Trampert 2010; Froment et al. 2010; Hanasoge 2013b; Fichtner 2014). Alternatives to Green's function retrieval have been developed in theory (e.g. Tromp et al. 2010; Hanasoge 2013b; Fichtner et al. 2017; Ermert et al. 2017; Sager et al. 2018b; Datta et al. 2019; Xu et al. 2019) and are reaching the level of being applicable to data (e.g. Sager et al. 2020; Xu et al. 2020). However, these methods require knowledge of the noise source distribution.

Methods to locate noise sources include beamforming with back projection (e.g. Gerstoft \& Tanimoto 2007; Juretzek \& Hadziioannou 2016; Retailleau et al. 2017; Retailleau \& Gualtieri 2019) or 
matched-field processing (e.g. Gal et al. 2018) which require different assumptions, such as plane wave propagation, or a sufficiently dense array. To properly represent ambient wave propagation through the 3-D Earth, several authors adopted the direct numerical modelling of noise correlations for heterogeneous noise sources (e.g. Nishida \& Fukao 2007; Tromp et al. 2010; Hanasoge 2013a; Ermert et al. 2017; Sager et al. 2018a; Datta et al. 2019; Xu et al. 2019), based on concepts originally developed in helioseismology (Woodard 1997; Gizon \& Birch 2002). This approach naturally yields noise source sensitivity kernels that may be used to infer the spatial distribution of sources, while honouring the physics of wave propagation through a 3-D heterogeneous medium (Ermert et al. 2017, 2020). Another approach that has been shown to be effective to model ambient noise correlations is normal mode summation (e.g. Nishida \& Fukao 2007; Gualtieri et al. 2013).

To reduce computational cost, especially when higher frequencies are involved, Ermert et al. (2017) proposed an implementation based on pre-computed wavefields from numerical wavefield solvers. This is particularly effective in an inversion framework, because the wavefield only has to be computed once. Due to the chosen measurement, namely the logarithmic energy ratio which is largely insensitive to unknown 3-D Earth structure (Sager et al. 2018a), we can justify using simpler 1-D models like PREM (Dziewonski \& Anderson 1981) as underlying velocity model.

We aim to efficiently forward model noise cross-correlations on a global scale for any noise source distribution for frequencies up to $0.2 \mathrm{~Hz}$. To achieve this, we implement a spatially variable grid alongside pre-computed wavefields to reduce the number of model parameters and thus the computational cost. We assess the accuracy of the reduced parameterisation by carrying out benchmark tests with a globally homogeneous parameterisation and a different wave propagation solver. Furthermore, the aforementioned sensitivity kernels allow us to easily implement a gradient-based iterative method to invert for the noise source distribution. Following a brief review of the underlying equations, we perform synthetic inversions to test the method under idealistic circumstances. Finally, we present real-data inversions for several consecutive days.

\section{FORWARD MODELLING}

The concept of modelling cross-correlations for arbitrary noise source distributions originated in helioseismology (Woodard 1997) and has been modified for the use on Earth by several authors (Tromp et al. 2010; Hanasoge 2013b; Fichtner 2014). To provide the necessary context, we give a short derivation of the cross-correlation wavefield equations, similar to Ermert et al. (2017, 2020). Subsequently, we describe our approach to make the computation feasible for global, high-frequency problems using pre-computed wavefields and spatially variable grids. 


\subsection{Continuous and discretised modelling equations}

The ambient noise field is a superposition of elastic waves excited by a distributed source $N_{n}(\boldsymbol{\xi}, \omega)$ with $n$-component where $\boldsymbol{\xi}$ denotes the location on the Earth's surface $\partial \oplus$. We henceforth omit the frequency dependency $\omega$ in the interest of condensed notation. The $i$-component of ground motion, $u_{i}(\boldsymbol{x})$, at receiver position $\boldsymbol{x}$ can therefore be expressed as a convolution of the Green's function $G_{i n}(\boldsymbol{x}, \boldsymbol{\xi})$ for some suitable Earth model and the noise sources $N_{n}(\boldsymbol{\xi})$ :

$$
u_{i}(\boldsymbol{x})=\int_{\partial \oplus} G_{i n}(\boldsymbol{x}, \boldsymbol{\xi}) N_{n}(\boldsymbol{\xi}) d \boldsymbol{\xi} .
$$

The Einstein summation convention for repeated indices is implied. The ensemble-averaged frequencydomain correlation of two wavefield recordings at positions $\boldsymbol{x}_{1}$ and $\boldsymbol{x}_{2}$ is given by

$$
\begin{aligned}
& \mathcal{C}_{i j}\left(\boldsymbol{x}_{1}, \boldsymbol{x}_{2}\right)=\left\langle u_{i}^{*}\left(\boldsymbol{x}_{1}\right) \cdot u_{j}\left(\boldsymbol{x}_{2}\right)\right\rangle \\
& =\left\langle\iint_{\partial \oplus} G_{i n}^{*}\left(\boldsymbol{x}_{1}, \boldsymbol{\xi}_{1}\right) G_{j m}\left(\boldsymbol{x}_{2}, \boldsymbol{\xi}_{2}\right) N_{n}^{*}\left(\boldsymbol{\xi}_{1}\right) N_{m}\left(\boldsymbol{\xi}_{2}\right) d \boldsymbol{\xi}_{1} d \boldsymbol{\xi}_{2}\right\rangle
\end{aligned}
$$

In Eq. (3), $\langle\cdot\rangle$ denotes the averaging over several time intervals and * the complex conjugate. Thus, to model a cross-correlation wavefield, we need to model the Green's functions $G_{i n}(\boldsymbol{x}, \boldsymbol{\xi})$ and parameterise the noise sources $N_{n}(\boldsymbol{\xi})$. The temporal averaging has to be sufficiently long to ensure that purely stochastic fluctuations (e.g., instrumental noise) become irrelevant compared to the ambient noise signal that we wish to exploit. For modern broadband seismometers, as used in the application part of this work, instrumental noise levels are typically insignificant compared to ambient noise levels, especially within the microseismic frequency band. It is therefore safe to assume that instrumental noise is practically irrelevant when averaging time scales are an order of magnitude longer than the longest period considered in the correlation analysis. In practice, the averaging length is more controlled by the need to obtain correlation time series that are sufficiently simple, e.g., close to a Green's function, in order to make intuitively interpretable measurements that allow us to formulate a well-behaved inverse problem.

The correlation of the two noise sources can be described by their location-dependent powerspectral density (PSD) $S_{n m}(\boldsymbol{\xi})$,

$$
\left\langle N_{n}^{*}\left(\boldsymbol{\xi}_{1}\right) N_{m}\left(\boldsymbol{\xi}_{2}\right)\right\rangle=S_{n m}\left(\boldsymbol{\xi}_{1}\right) \delta\left(\boldsymbol{\xi}_{1}-\boldsymbol{\xi}_{2}\right)
$$

where we made the common assumption that neighbouring noise sources at $\boldsymbol{\xi}_{1}$ and $\boldsymbol{\xi}_{2}$ are temporally 
uncorrelated (e.g. Tromp et al. 2010; Hanasoge 2013b; Fichtner 2014). Upon inserting (4) into (3) the ensemble correlation condenses to

$$
\mathcal{C}_{i j}\left(\boldsymbol{x}_{1}, \boldsymbol{x}_{2}\right)=\int_{\partial \oplus} G_{i n}^{*}\left(\boldsymbol{x}_{1}, \boldsymbol{\xi}\right) G_{j m}\left(\boldsymbol{x}_{2}, \boldsymbol{\xi}\right) S_{n m}(\boldsymbol{\xi}) d \boldsymbol{\xi} .
$$

Eq. (5) allows us to evaluate a cross-correlation wavefield $\mathcal{C}_{i j}$ at position $\boldsymbol{x}_{1}$ with a reference station at position $\boldsymbol{x}_{2}$ for any noise source distribution $S_{n m}(\boldsymbol{\xi})$.

\subsection{Pre-computed Wavefields}

Since we are solely interested in the noise source distribution $S_{n m}(\boldsymbol{\xi})$ and not in changes of Earth structure, we can take advantage of pre-computed Green's function databases to reduce the computational cost of the correlation wavefield modelling. This approach can be further justified by choosing a suitable correlation waveform measurement that is largely insensitive to unknown 3-D Earth structure. This topic will be discussed in more detail in section 3 .

Ambient noise propagation is an inherently global phenomenon, even when stations used for data analysis cluster at regional scale (e.g. Hillers et al. 2012; Retailleau et al. 2017; Sager et al. 2018a). Several wave propagation solvers have been developed for global waye simulations - for instance, the finite-difference implementation of Igel \& Weber (1996), SpecFEM (Komatitsch \& Tromp 2002), AxiSEM (Nissen-Meyer et al. 2014), and more recently Salvus (Afanasiev et al. 2019) - which make the computation of global wavefields an attainable task when sufficient computational resources are available. Since this may not be the case, especially at higher frequencies, some databases are shared online by, for example, the IRIS Synthetics Engine (Syngine) repository (IRIS 2015; Krischer et al. 2017) which gives access to global wavefield databases computed with AxiSEM (Nissen-Meyer et al. 2014) in 1-D Earth models. From pre-computed AxiSEM wavefields, we can easily extract seismograms using the Python package Instaseis (van Driel et al. 2015).

To model ambient noise we compute a Green's function database with the help of reciprocity where each receiver is taken as a point source and the corresponding seismograms are recorded at all grid points. We then approximate the frequency-domain correlation wavefield by a sum over Green's functions multiplied with their corresponding noise source weight $S\left(\boldsymbol{\xi}_{k}\right)$,

$$
\mathcal{C}_{i j}\left(\boldsymbol{x}_{1}, \boldsymbol{x}_{2}\right) \approx \sum_{k=1}^{n_{g}} G_{i n}^{*}\left(\boldsymbol{x}_{1}, \boldsymbol{\xi}_{k}\right) G_{j m}\left(\boldsymbol{x}_{2}, \boldsymbol{\xi}_{k}\right) S_{n m}\left(\boldsymbol{\xi}_{k}\right) \Delta \boldsymbol{\xi}_{k},
$$

where $\Delta \boldsymbol{\xi}_{k}$ is an approximation of the finite surface element, and $n_{g}$ is the number of grid points (discrete noise sources). The computational cost of (6) scales with the number of grid points, suggesting 
that the noise source parameterisation should be optimised for efficient noise correlation modelling. A possible optimisation is the topic of the following section.

\subsection{Spatially Variable Grid}

We reduce the number of grid points by implementing a spatially variable grid. This is possible thanks to the shape of the noise source sensitivity kernels, which become broader linearly with increasing distance from the station pair (Hanasoge 2013b), thereby requiring less spatial resolution for the parameterisation. Furthermore, attenuation causes distant noise sources to be of lesser importance, additionally avoiding the refocusing of sensitivity at station antipoles. To obtain a parameterisation that is both flexible and simple, we implement spatially variable Gaussian grids, some examples of which are visualised in Fig. 1. The radial distance $d \phi$ between concentric circles of grid points on a sphere is described by

$$
d \phi= \begin{cases}d \phi_{\min } & \text { if } \phi<\sigma \\ d \phi_{\min }+d \phi_{\max } *\left(1-e^{-i^{\beta}}\right) & \text { else, }\end{cases}
$$

where the minimum and maximum incremental grid point distance is represented by $d \phi_{\min }$ and $d \phi_{\max }$, the radius of the area of high grid point density by $\sigma$, and the steepness of the increase in grid point distance by $\beta$. The iteratively increasing variable $i$, representing the index of each circle, is used to create the distribution up to the point where the sum of all $d \phi$ is $180^{\circ}$, that is, the whole globe is accounted for by the concentric radial circles. We set the distance between grid points on each circle to roughly the radial distance, to ensure approximate homogeneity of the grid. Additionally, the geographical centre of the grid can be shifted to any location.

Fig. 1 shows the radial grid point distance from the centre of the concentric circles for different values of $\sigma$ and $\beta$. For $\phi<\sigma$ the function is constant to ensure that the grid is nearly homogeneous up to a distance of $\sigma$ from the centre. Subsequently, a modified Gaussian takes over and increases the distance at an adjustable rate $\beta$ up to a constant grid point distance $d \phi_{\max }$. Increasing $\sigma$ leads to a larger area of dense grid points around the geographical centre of the concentric circles. The parameter $\beta$ adjusts the steepness of the modified Gaussian function; a larger value means the grid point distance increases more rapidly with distance from the dense region and vice versa. The parameters are chosen on a case-by-case basis depending on the area of interest, station locations, frequency range, and available computational resources.

We implement further options to increase adaptivity to heterogeneous noise sources. To account for multiple strong noise sources, we allow multiple areas with higher spatial resolution. This is achieved with a coarse background grid from which grid points are removed, and smaller-scale denser 


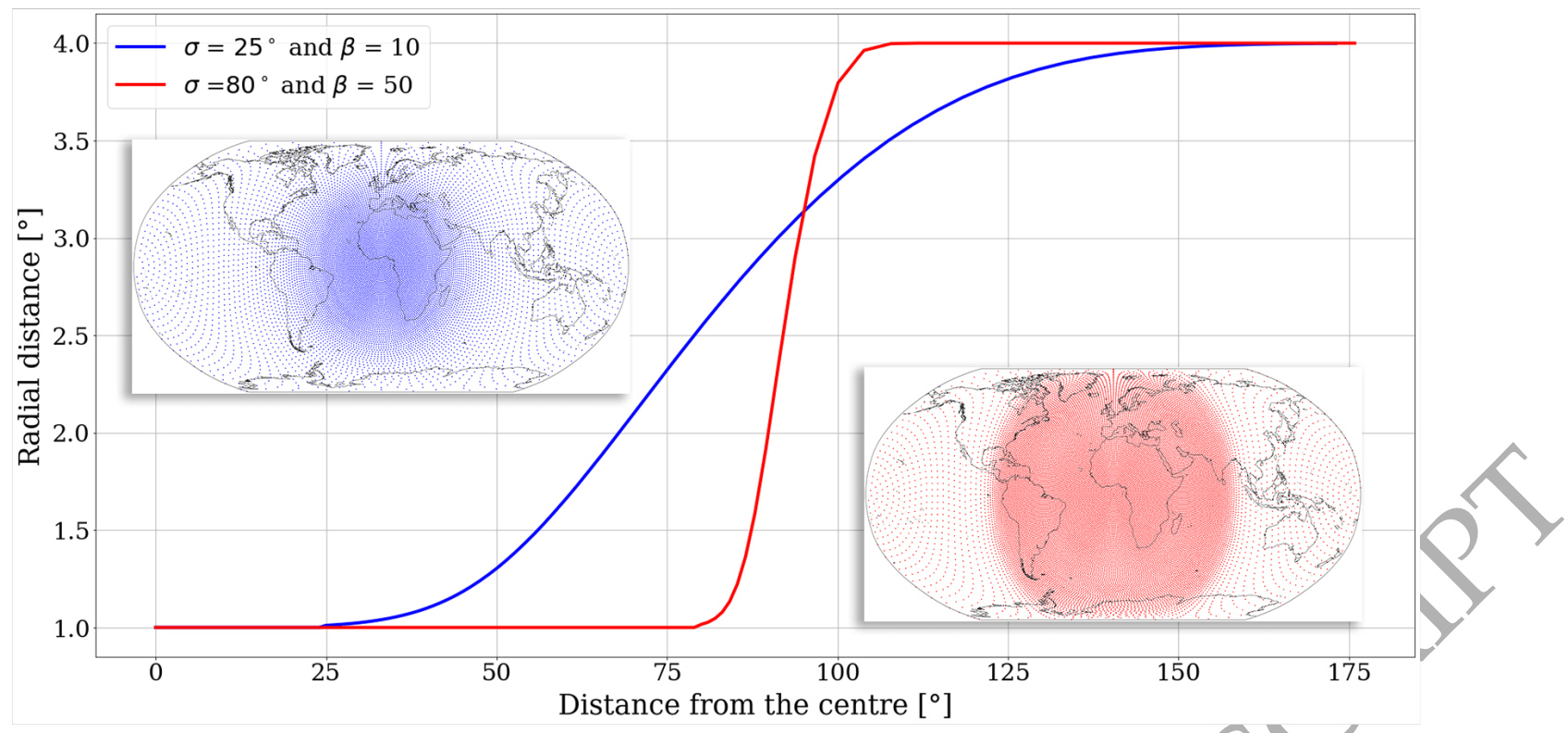

Figure 1. Two spatially variable grids with different parameters for the radius of the high density area $\sigma\left[{ }^{\circ}\right]$ and steepness of the increase in grid point distance $\beta$. Radial distance is the distance between the concentric circles, and the distance from the centre corresponds to the cumulative sum of all previous radial distances.

grids added. If prior information about the noise source distribution is available (for instance from ocean surface pressure maps that are related to secondary microseimic sources, see Ardhuin et al. 2011) the spatial resolution can be increased in areas of higher PSD. We choose the threshold value of the PSD above which additional sub-grids with higher spatial resolution are added in a given radius around the sources. This ensures that the structure of the strong noise sources is considered, whereas areas of weak noise sources have a sparser grid to save computational cost. An example of such an adapted grid is shown in Fig. 2. Here, the input model used for correlation computations is an ocean surface pressure map by Ardhuin et al. (2011).

To obtain suitable surface elements $\Delta \boldsymbol{\xi}_{k}$ for spatially variable grids, we compute the Voronoi cell surface area for each grid point using the Python package SciPy (Virtanen et al. 2020). This ensures that an accumulation of grid points does not lead to stronger noise sources in that particular area.

Combining pre-computed wavefields and spatially variable grids enables us to efficiently model global cross-correlations for any given noise distribution.

\subsection{Benchmarks}

To quantify the potential computational savings of the adaptive gridding, we compare correlations computed with spatially variable and globally homogeneous grids. Considering only oceanic sources, the total number of points ranges from 1,000 to 80,000. Since the 24 stations selected for the bench- 


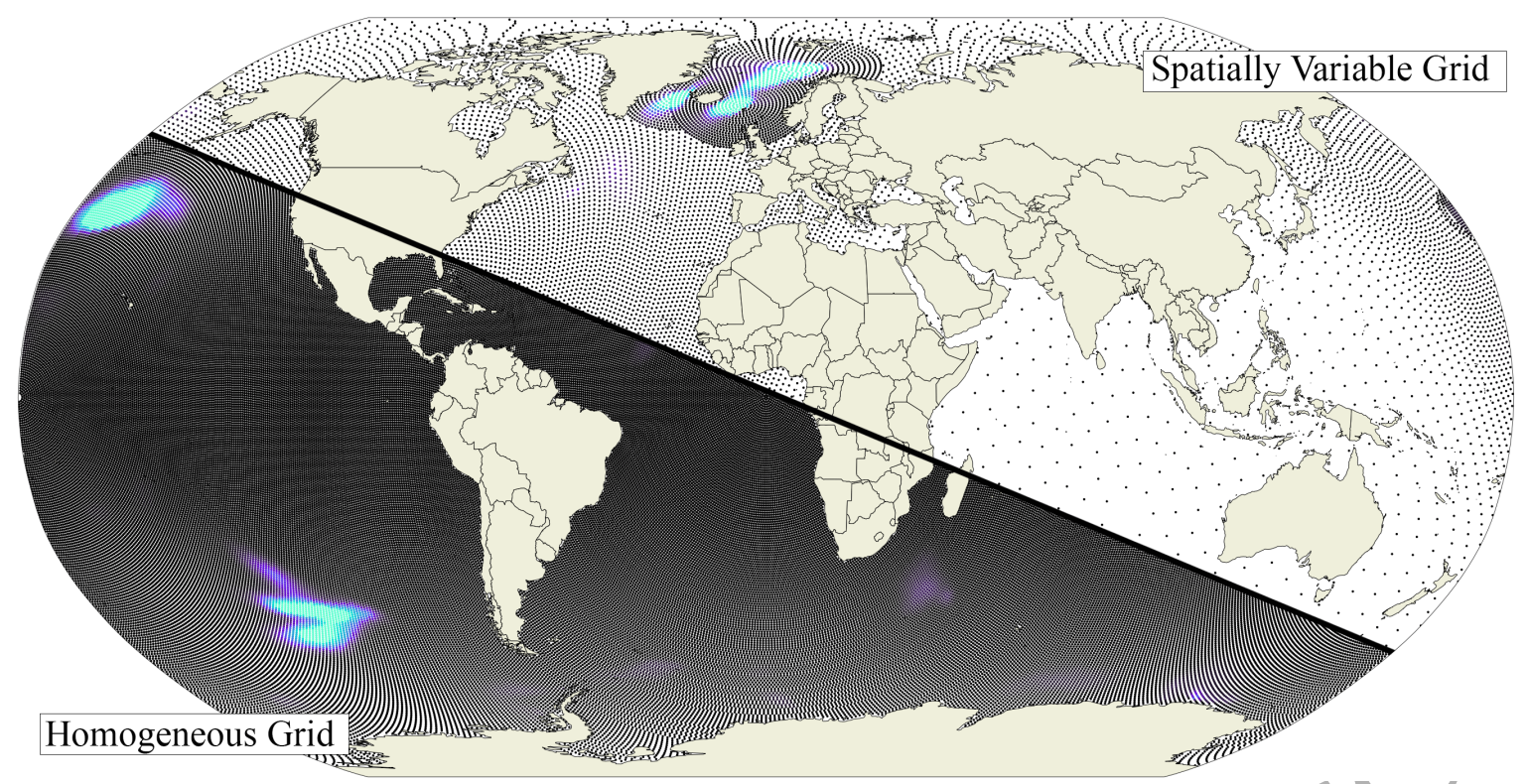

Figure 2. Comparison of a homogeneous grid (bottom left) and an automatically generated spatially variable grid (top right) where high PSD areas (bright blue) have a higher spatial resolution. The grid point distance of the homogeneous grid (120,000 grid points) is equal to the grid point distance of the highest density areas of the spatially variable grid (19,000 grid points).

marks are located around the North Atlantic, we increase resolution of the spatially variable grid in that region. Two example grids for roughly 20,000 grid points and the station locations can be seen in Fig. 3. The 24 stations yield 276 cross-correlations which are compared to reference cross-correlations computed with a homogeneous grid of 100,000 points. We pre-compute the Green's function database using AxiSEM (Nissen-Meyer et al. 2014) for frequencies up to $0.1 \mathrm{~Hz}$. The underlying Earth model is PREM (Dziewonski \& Anderson 1981)

As a measure of correlation waveform fit we employ, in the interest of simplicity, the $L_{2}$ distance between the correlations computed using the test grids $C_{i}^{\text {test }}$ and the reference grid $C_{i}^{\text {ref }}$ :

$$
\chi_{\text {grid }}=\frac{1}{N} \sum_{i=1}^{N} \int\left[C_{i}^{r e f}(t)-C_{i}^{t e s t}(t)\right]^{2} d t
$$

where $N$ denotes the number of station pairs. Fig. 3 shows how the $L_{2}$ distance evolves with an increasing number of grid points. The spatially variable grid initially has a lower misfit and also converges faster than the homogeneous grid. For instance, for the homogeneous grid we require around 12,000 grid points to reach a misfit of 0.1 , whereas the spatially variable grid only requires 4,000 grid points. Hence, in this case, we may reduce the number of model parameters by a factor of 3 and still expect results to be nearly identical. Depending on the chosen threshold, this parameter reduction varies between 2.5 and 4.5 . 


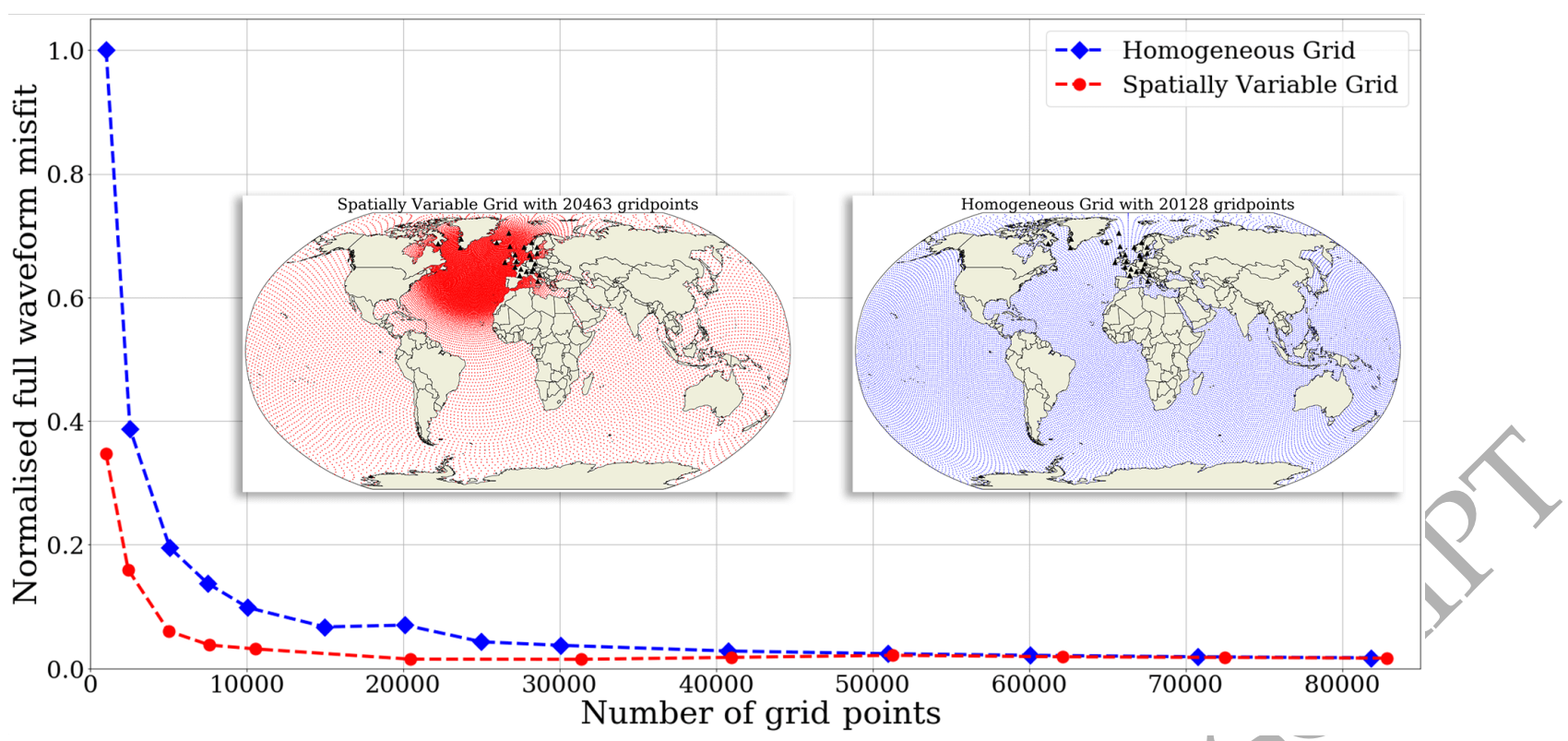

Figure 3. Benchmarks for spatially variable and globally homogeneous grids. The inséts show two example grids, with station locations marked by black triangles. The $L_{2}$ distance is calculated with respect to reference correlations computed with a homogeneous grid of 100,000 points. To attain a misfit of 0.1 we require 12,000 grid points for a homogeneous grid but only 4,000 grid points with a spatially variable grid, meaning that we may reduce the number of grid points by a factor of 3 .

While this example illustrates the potential of the variable grid approach, its efficiency is clearly application-specific and needs to be assessed carefully on a case-by-case basis prior to a large-scale inversion that requires numerous forward simulations. Particularly global inversions poorly benefit from such a spatially variable grid as most areas are high-sensitivity regions. However, the grid could still be adapted if strong noise source areas are known beforehand.

An alternative to the direct discretisation of Eq. (6) combined with a Green's function database is on-the-fly computation of the correlation wavefield source $G_{j m}\left(\boldsymbol{x}_{2}, \boldsymbol{\xi}\right) S_{n m}(\boldsymbol{\xi})$ on the grid of a numerical wave propagation solver, and its use in a subsequent forward simulation (Tromp et al. 2010; Fichtner 2014; Sager et al. 2020). This approach trades the larger storage requirements of the Green's function database for larger computational requirements in an iterative inversion, where correlations must be computed for a sequence of noise source updates. Comparing these independent approaches provides another useful plausibility check on the quality of the correlation functions. This is described in more detail in supplementary section S1. 


\section{MEASUREMENTS AND SENSITIVITY KERNELS}

To constrain the distribution of noise sources, we require information on how changes in the noise source PSD, $S_{n m}(\boldsymbol{\xi})$, affect the fit between observed and synthetic correlations. Applying adjoint techniques allows us to reduce the computational cost to obtain the source sensitivity kernels by using the pre-computed Green's function databases. More details and in-depth derivations for finite-frequency sensitivity kernels can be found in Tromp et al. (2010), Fichtner (2014), Ermert et al. (2017), Hanasoge (2013b) or Sager et al. (2018a). The final expression for a source sensitivity kernel for the components $i$ and $j$ of a station pair with source PSD components $n, m$ is

$$
K_{n m}\left(\boldsymbol{x}_{1}, \boldsymbol{x}_{2}, \boldsymbol{\xi}\right)=\int G_{i n}\left(\boldsymbol{x}_{1}, \boldsymbol{\xi}\right)^{*} G_{j m}\left(\boldsymbol{x}_{2}, \boldsymbol{\xi}\right) f_{i j}\left(\boldsymbol{x}_{1}, \boldsymbol{x}_{2}\right) d \omega,
$$

where $f_{i j}\left(\boldsymbol{x}_{1}, \boldsymbol{x}_{2}\right)$ is the measurement-dependent adjoint source which excites the adjoint wavefield at the receiver position $\boldsymbol{x}_{1}$. Since we have already pre-computed the Green's function database, the kernel computation does not require any additional simulations.

While there are numerous possible measurements that may be used to quantify differences between observed and synthetic correlation waveforms, we limit ourselves to the causal-acausal logarithmic energy ratio, defined as

$$
A=\ln \left(\frac{\int[w(\tau) C(\tau)]^{2} d \tau}{\int[w(-\tau) C(\tau)]^{2} d \tau}\right)=\ln \left(\frac{E_{+}}{E_{-}}\right),
$$

with the time window $w(\tau)$ at lag $\tau$ centred around the expected surface-wave arrival time of the correlation $C(\tau)$. The observed energy ratio $A^{\text {obs }}$ is defined accordingly. The outstanding advantage of the asymmetry measure $A$ is its relative insensitivity to unknown 3-D Earth structure (Sager et al. 2018a), which adds another justification to the pre-computed wavefield approach where we keep the Green's functions constant during the inversion process. Discrepancies in the surface wave arrival times due to unknown 3-D Earth structure can be counteracted by increasing the expected surface wave arrival time window in the measurement. This ensures that the energy calculation still includes the main wavelets even when the given surface wave velocity is not entirely accurate. Due to this characteristic of the measurement we currently compute the Green's functions using the 1-D PREM (Dziewonski \& Anderson 1981) as underlying velocity model.

Based on Eq. (10), we define the $L_{2}$ misfit as a sum over asymmetry differences measured at all station pairs,

$$
\chi=\frac{1}{2} \sum_{i=1}^{N}\left[A_{i}-A_{i}^{o b s}\right]^{2} .
$$




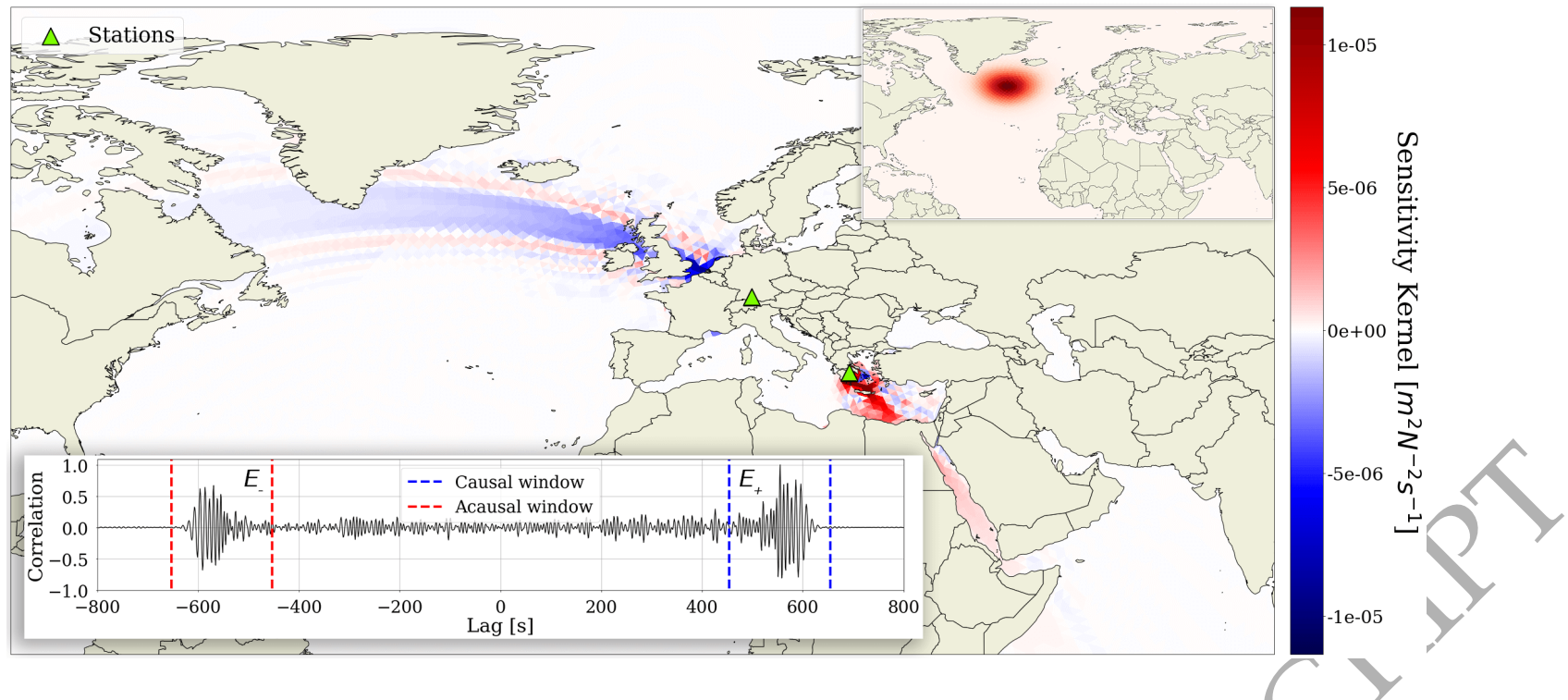

Figure 4. Illustration of signal energy measurements on the causal and acausal branch of a synthetic crosscorrelation and the corresponding sensitivity kernel. A $100 \mathrm{~s}$ time window around the expected surface-wave arrival time with a wave velocity of $2.8 \mathrm{~km} / \mathrm{s}$ is used for the measurement. Artificial observed data are for a dominant noise source in the North Atlantic south of Iceland (top right inset), whereas synthetic correlations are based on a homogeneous noise distribution in the oceans. Negative sensitivity implies that an increase in source PSD will decrease the misfit.

Finally, we derive the equation for the measurement-dependent adjoint source from Eq. 10 and Eq. 11 to obtain

$$
f(\omega)=\left(A-A^{o b s}\right)\left[\frac{1}{\pi E_{+}}\left(w_{+}^{2}(\omega) * C(\omega)\right)^{*}-\frac{1}{\pi E_{-}}\left(w_{-}^{2}(\omega) * C(\omega)\right)^{*}\right] .
$$

A more detailed derivation of the adjoint source for this specific choice of measurement and misfit can be found in Ermert et al. (2016).

An illustration of the signal energy measurement and the corresponding sensitivity kernel can be seen in Fig. 4. To obtain the example sensitivity kernel we computed an artificial observed correlation for one station pair with a dominant noise source south of Iceland. The synthetic cross-correlation is based on a homogeneous noise distribution in the oceans. As expected, the sensitivity kernel is largely negative in the North Atlantic, suggesting that an increase in noise source PSD in that region will decrease the misfit.

\section{MICROSEISMIC NOISE SOURCE INVERSION}

We perform several synthetic and real-data ambient noise source inversions using vertical-verticalcomponent correlations and a gradient-based iterative scheme to minimise the misfit in Eq. (11), sim- 
ilar to the method applied to the Earth's hum by Ermert et al. (2017). Since vertical-component data mostly constrain vertical-component sources, we restrict all inversions to $S_{z z}$, setting the remaining components of the PSD tensor to 0. Prior to showing results we describe the overall setup, which is the same for both synthetic and real-data applications.

The underlying Green's function database is the result of AxiSEM (Nissen-Meyer et al. 2014) simulations with frequencies up to $0.2 \mathrm{~Hz}$. This allows us to invert for secondary microseismic sources between 0.1 and $0.2 \mathrm{~Hz}$. Velocity, attenuation, and density structure are based on PREM (Dziewonski \& Anderson 1981), and the noise source model is parameterised using a spatially variable grid with denser grid point spacing in the North Atlantic. Due to the shape of source sensitivity kernels, it is beneficial to use stations around the area of interest to obtain good coverage. Thus, we choose stations surrounding the North Atlantic in North America and Europe.

An illustration of ray coverage and station sensitivity for a set of station locations is presented in Fig. 5. The station sensitivity is computed as the sum of absolute values of all sensitivity kernels, and serves as a proxy for regions where noise source PSD may be constrained. To avoid local sensitivity peaks from dense arrays, we set a minimum station distance of $1^{\circ}$.

To further decrease the computational cost, we choose the observed cross-correlations for the inversion based on a modified signal-to-noise ratio. This is computed by dividing the maximum amplitude in the expected arrival time windows by the standard deviation of the whole time series (see Fig. S2 for visualisation). Since the computational cost directly scales with the number of station pairs, we set a minimum signal-to-noise threshold of 3 to filter out cross-correlations with little information in the surface wave arrival time windows. This is usually the case if the dominant noise sources are not in-line with the station pair.

Since the secondary microseisms are solely generated by ocean waves, we take a spatially homogeneous noise distribution in the oceans as the initial model. We parameterise the noise sources using a spatially variable grid with roughly 14,000 grid points, removing all grid points on land. Since our stations surround the North Atlantic we increase the spatial resolution there. Furthermore, we parameterise the frequency spectrum of each noise source by the peak amplitude of a Gaussian with centre frequency of $0.15 \mathrm{~Hz}$ and standard deviation of $0.05 \mathrm{~Hz}$. Observed and synthetic correlations are filtered accordingly.

To minimise the asymmetry misfit, we employ an iterative steepest-descent method, where correlations, misfits, and kernels are re-computed for every model update. Prior to any update, we apply widely used preconditioning, including 95th percentile clipping and Gaussian smoothing of the kernels. The standard deviation of the Gaussian smoothing filter decreases during the inversion from $4^{\circ}$ to $1.5^{\circ}$ in order to initially avoid local minima and later permit more spatial detail. These values are 


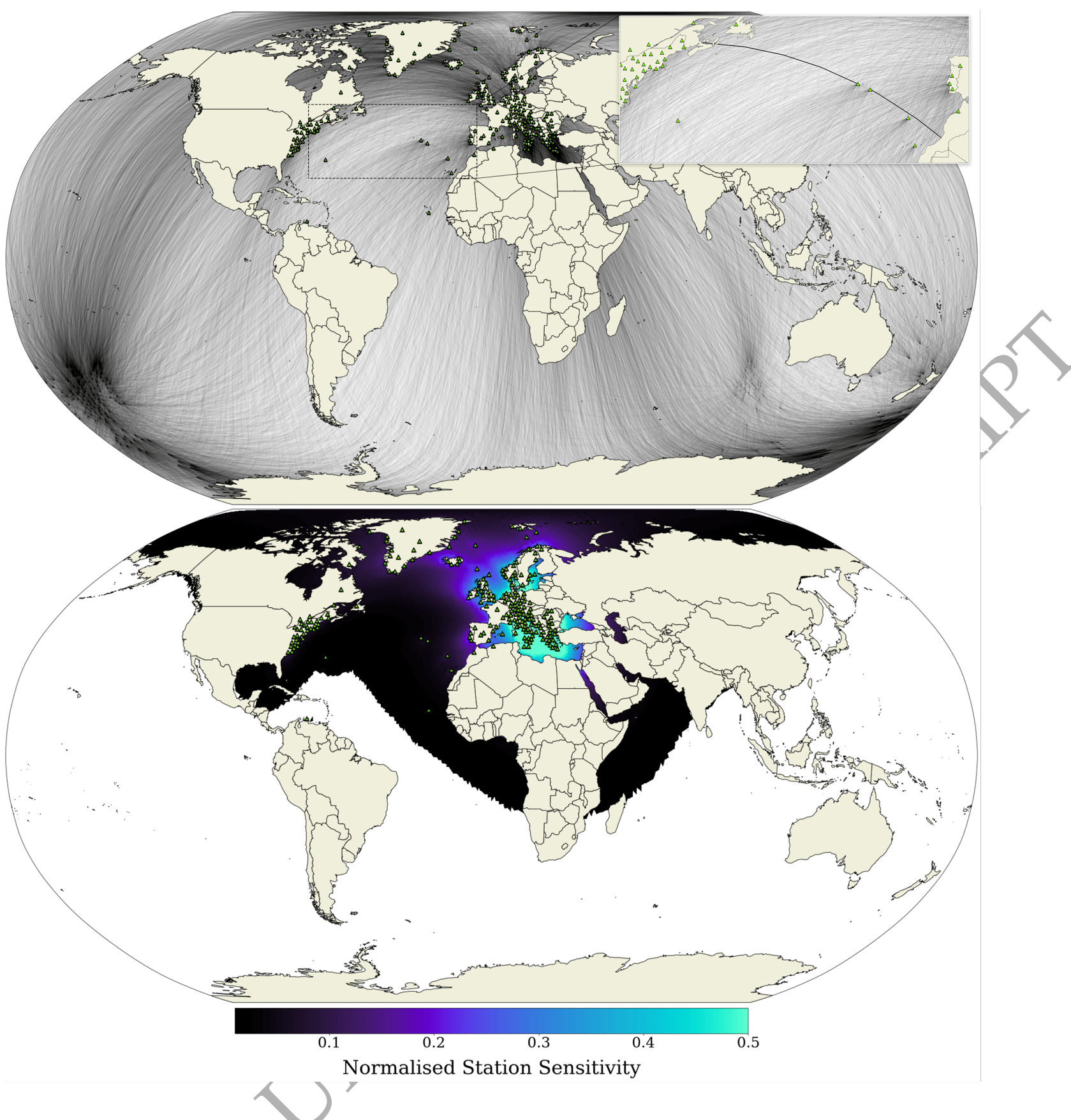

Figure 5. Ray coverage (top) and station sensitivity (bottom) for a set of station locations (green triangles) surrounding the Northern Atlantic. Due to the shape of the sensitivity kernels, the rays are not between but behind the station pair (top right inset). The station sensitivity is computed by taking the sum of the absolute values of all sensitivity kernels. Regions where station sensitivity is below $1 \%$ of the maximum are masked in order to indicate that information to constrain noise sources is likely to be insufficient. Since we take attenuation into account, station sensitivity does not refocus at the station antipoles, in contrast to the simplified rays shown in the top panel. 
largely the result of trial and error based on a series of synthetic inversions. Since PSD cannot be negative, we introduce a non-negativity constraint by setting all sources with negative PSD to 0 after each model update.

In the following paragraphs we present synthetic inversions intended to test the inversion scheme for different noise distributions and station locations under idealised conditions. Furthermore, we perform real-data inversions for several consecutive days.

\subsection{Synthetic inversions}

As a first synthetic application of the inversion method we use a significant wave height map from the WaveWatch III model (Tolman \& Chalikov 1996) as the input noise source PSD distribution for the computation of artificial observed correlations. We choose 217 stations surrounding the North Atlantic to perform the inversion, resulting in roughly 12,500 measurements excluding all correlations with a signal-to-noise ratio below 3 . Executing the approach outlined above, the misfit drops by $\approx 75 \%$ after the first iteration, and by $92 \%$ after ten iterations. The observation that the misfit plateaus quickly is typical also for other inversions presented later, and it leads us to generally stop the misfit minimisation after 10 iterations.

A summary of this first inversion is presented in Fig. 6, where we do not plot areas where the station sensitivity (seen in Fig. 5) is below 1\%. Comparing the artificial distribution with the final inversion model, we see that the dominant noise sources off the coasts of Ireland, France and Spain can be recovered. Additionally, the spatially smaller sources on the southern coast of Greenland and between Iceland and Norway are included, even though the spatial resolution is evidently limited by the station geometry.

Similar to the first inversion, we use data from ocean surface pressure maps by Ardhuin et al. (2011) as input noise distribution for a second synthetic test. As can be seen in Fig. 7, these maps have smaller-scale noise sources compared to the significant wave height maps. Though the inversion is, as expected, unable to reselve such small details, it provides a qualitative assessment of the smearing length scales in our region of interest. After 10 iterations and a misfit reduction of $84 \%$, the noise sources east of Iceland and north of Scandinavia can be distinguished, whereas other features are more difficult to discern. Some small inversion artifacts due to the parameterisation of the noise sources i.e. lack of grid points on land - are visible in some areas, e.g. Barents Sea and Caspian Sea.

\subsection{Real-data inversions}

Finally, we apply our method to real-data correlations of ambient noise in order to infer the spatiotemporal behaviour of secondary microseism sources. To download and cross-correlate the noise data 


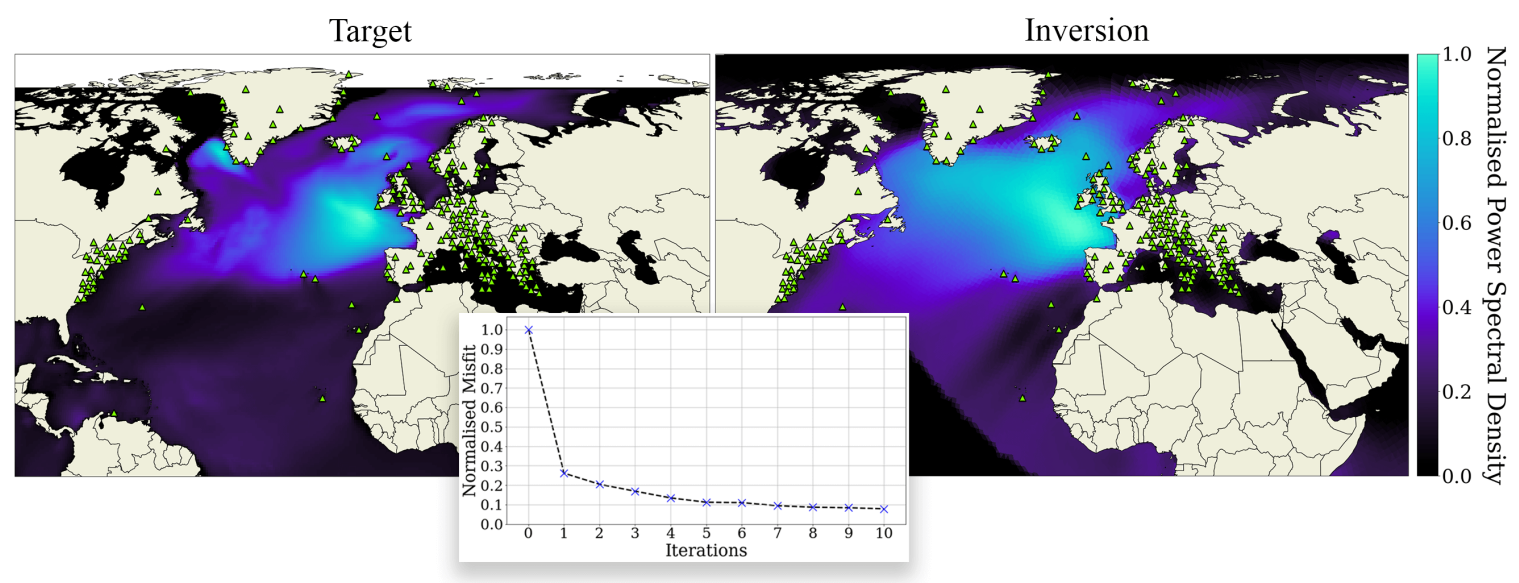

Figure 6. Summary of a synthetic inversion using WaveWatch III (left panel, Tolman \& Chalikov (1996)) as input for the computation of artificial observations. At the 10th iteration a misfit reduction of $92 \%$ is achieved. The dominant noise sources from the input distribution are included in the final inversion model, shown in the right panel. These include noise sources off the coast of Ireland, France, and Spain, as wellas spatially smaller sources on the south coast of Greenland and east of Iceland. The colourbar applies to both plots. The station locations are represented by green triangles.

we use the mass downloader from the Python-based toolbox ObsPy (Krischer et al. 2015). Motivated by the presence of Hurricane Lorenzo in the North Atlantic, we choose the first six days of October 2019 as time frame. Furthermore, there were no notable earthquakes during that time period, thereby allowing us to apply only basic linear processing that avoids potentially nonphysical effects (Fichtner

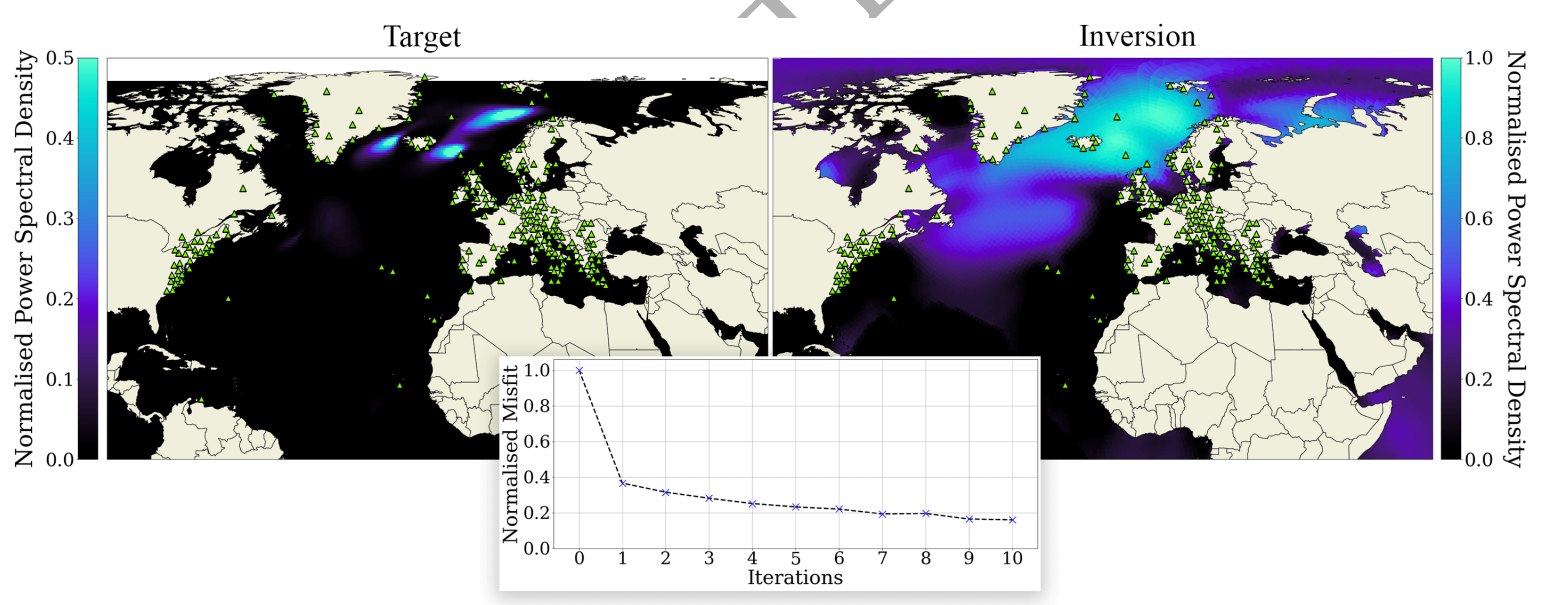

Figure 7, Summary of a synthetic inversion using an ocean surface pressure map by Ardhuin et al. (2011) as input for the computation of artificial observations. The misfit is reduced by $84 \%$. All three dominant noise sources in the ocean around Iceland are included in the final model. The left colourbar is capped at 0.5 to better show the ocean surface pressure distribution. The station locations are represented by green triangles. 
et al. 2020). First, we segment the data into 1-hour windows and remove the instrument response to obtain true displacement data. Subsequently, we downsample the data to $1 \mathrm{~Hz}$ and compute daily correlations by stacking individual correlations of the 24 time windows. Once the observed correlations are computed, we perform the inversions for six consecutive days.

Since we only use one day of ambient noise data, the signal-to-noise ratio in the correlations is small, particularly compared to the synthetic correlations. To ensure that we are actually inverting for a signal and not just a random time series, we ignore correlations with a signal-to-noise ratio below 3. Additionally, we add random noise to the synthetic correlations during the inversion to introduce a water level for the measurement which ensures we do not have 0 energy in a window and make the synthetics resemble observed data more closely. The noise level added is based on the maximum amplitude of each individual correlation and is kept constant throughout the inversion. Synthetic tests (see Fig. S6 and Fig. S7) show that this approach considerably improves the inversion model if the signal-to-noise ratio in the observed data is low. More analysis of different noise levels for the synthetic correlations and its effect on the inversion framework can be found in the supplementary material.

The final inversion models after 10 iterations are summarised in Fig. 8, where the models are normalised with the maximum PSD of the 6 day period. The models show the spatio-temporal variations of the source distribution of secondary microseisms in the wider North Atlantic region. The average misfit reduction is approximately $28 \%$.

Theoretically, the ocean surface pressure maps by Ardhuin et al. (2015) should correspond to the sources of the secondary microseisms when the ocean site effect is taken into account (LonguetHiggins 1950; Gualtieri et al. 2013, 2014; Ardhuin et al. 2019). Fig. 9 shows these maps for the six consecutive days in the same frequency range of $0.1-0.2 \mathrm{~Hz}$, with the same smoothing filter applied as for the noise correlation inversion. In these maps, the path of Hurricane Lorenzo is visible as it moves north towards Greenland.

Comparing the final inversion models with the ocean surface pressure maps, we observe that the areas of dominant noise sources are similar throughout the 6 day period. The source strengths in the North Atlantic gradually increase until the 4th October 2019. This corresponds to the day that Hurricane Lorenzo dissipates, after which the source strengths start to decrease again. We also observe some dominant noise sources in marginal seas like the Caspian Sea. However, we hypothesise that these sources have no real physical meaning as they originate from the parameterisation of the inverse problem, Correlations with a low asymmetry ratio correspond to a homogeneous noise distribution, i.e. the same source strength on both sides. Due to the lack of grid points on land this results in the sources in the marginal seas to overcompensate with very high source strengths.

A representative collection of correlations is shown in Fig. 10 for October 4, 2019. Synthetic cor- 

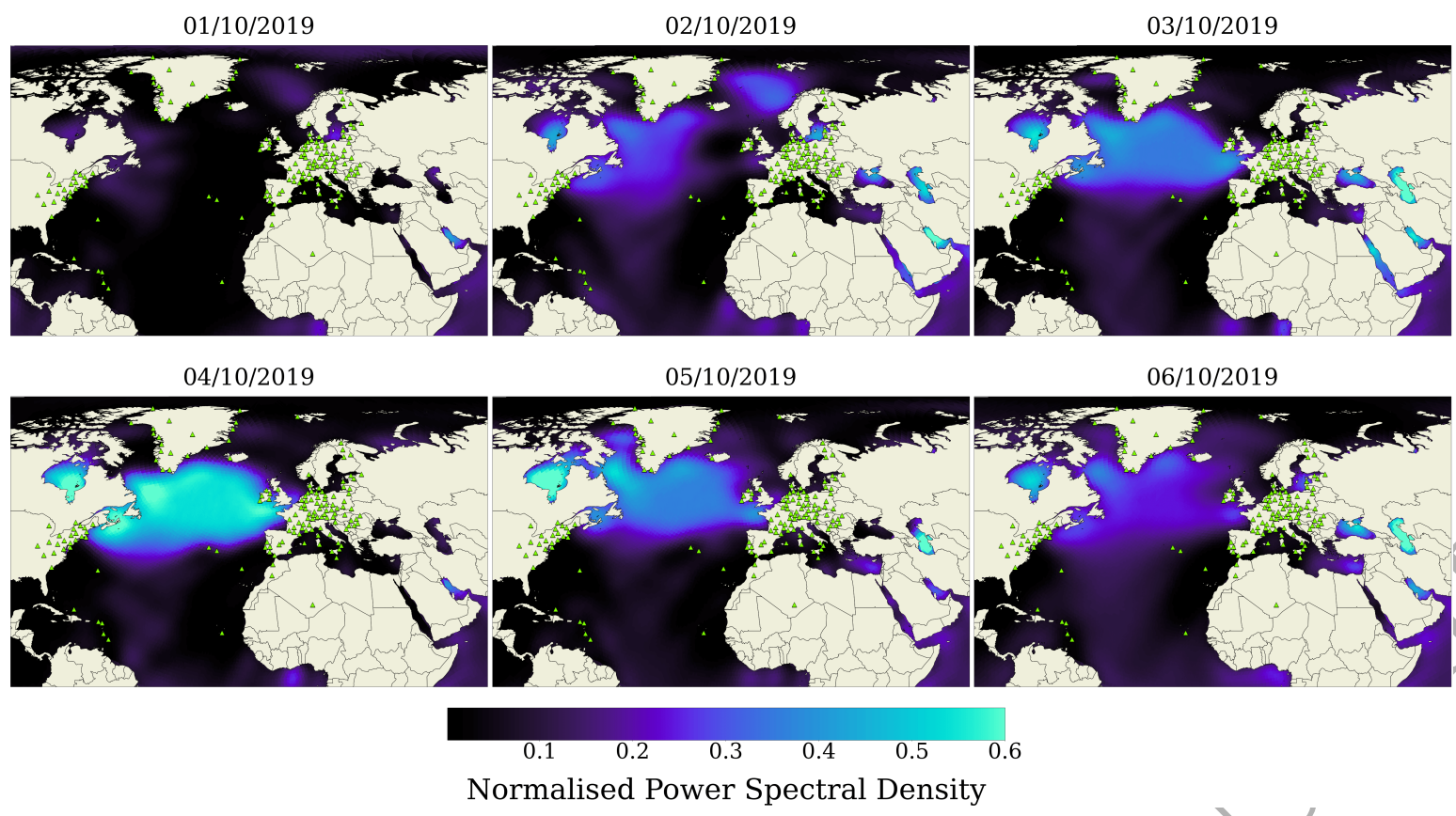

Figure 8. Noise source PSD models for the first six days of October 2019 after 10 iterations. The strong spatiotemporal variations of the sources of secondary microseisms are visible. The colourbar is capped at 0.6 for better visibility.
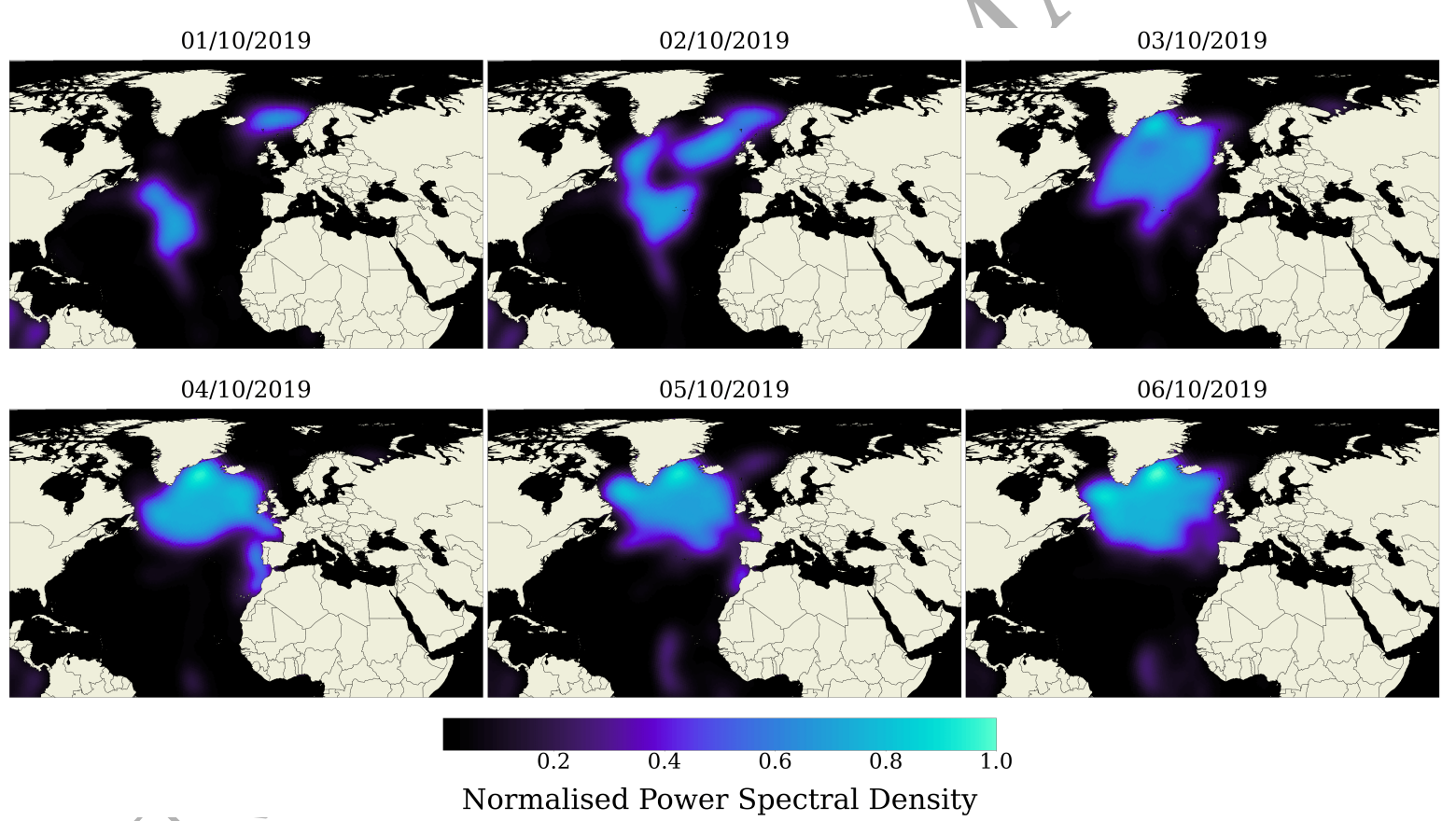

Figure 9. Ocean surface pressure maps including source site effect (Ardhuin et al. 2011; Longuet-Higgins 1950; Gualtieri et al. 2013) for the first six days of October 2019. Theoretically, these correspond to the sources of secondary microseisms. 
relations are shown for both the homogeneous initial model and the final model after 10 iterations. For stations pairs such as LD.UNH - LD.WADE, where the correlation resembles a plausible (one-sided) Green's function, the final synthetic correlation approximately explains the waveform of the observed correlations. Other station pairs close to the lower limit of the signal-to-noise ratio limit, for example CZ.MORC - WM.MAHO, do not contain clear surface wave arrivals. Since the asymmetry misfit only evaluates energy within the expected arrival time window and not the complete correlation waveform, the method is unable to fit the correlation waveform itself. Instead, it attempts to match causal and acausal energy, which contains less information but is more robust. Correlations with this lack of asymmetry automatically have less weight as the measurement is small compared to correlations with a clear asymmetry. By adding noise to the synthetics we avoid big discrepancies between the observed and synthetic measurements which would cause unwanted effects due to the parameterisation of the noise sources.

\section{DISCUSSION}

In the previous sections, we presented a global-scale finite-frequency ambient noise source inversion in the secondary microseismic frequency range. This is made possible by optimising the parameterisation and reusing pre-computed Green's function to greatly reduce the computational cost. Compared to other studies, we are able to circumvent the most common assumptions like the reduction to simple rays, the quasi-random nature of noise sources, and the plane wave assumption commonly used in beamforming, by using finite-frequency sensitivity kernels. By doing so, we properly account for global wave propagation with visco-elastic attenuation. Additionally, we only apply linear processing steps to the data reducing the risk of numerical artifacts in the cross-correlations. Thus, this method can be applied to any existing, linearly processed ambient noise cross-correlation data set. In the following, we provide a more detailed discussion on several key issues, such as measurements, 3-D structure effects, and the effective nature of the results.

\subsection{Measurements and time windows}

Our measurement, logarithmic energy ratio, relies on the existence of surface wave arrivals in the expected time window. However, when the dominant noise sources are not in-line with the receiver pair, spurious (but still physically meaningful) arrivals may appear outside the expected time window. These spurious arrivals are not taken into account so far. A more flexible measurement window, e.g., where we correlate for the time shift as well, could further improve the method and contribute more measurements to the inversion. Additionally, we only consider the vertical component of ground 

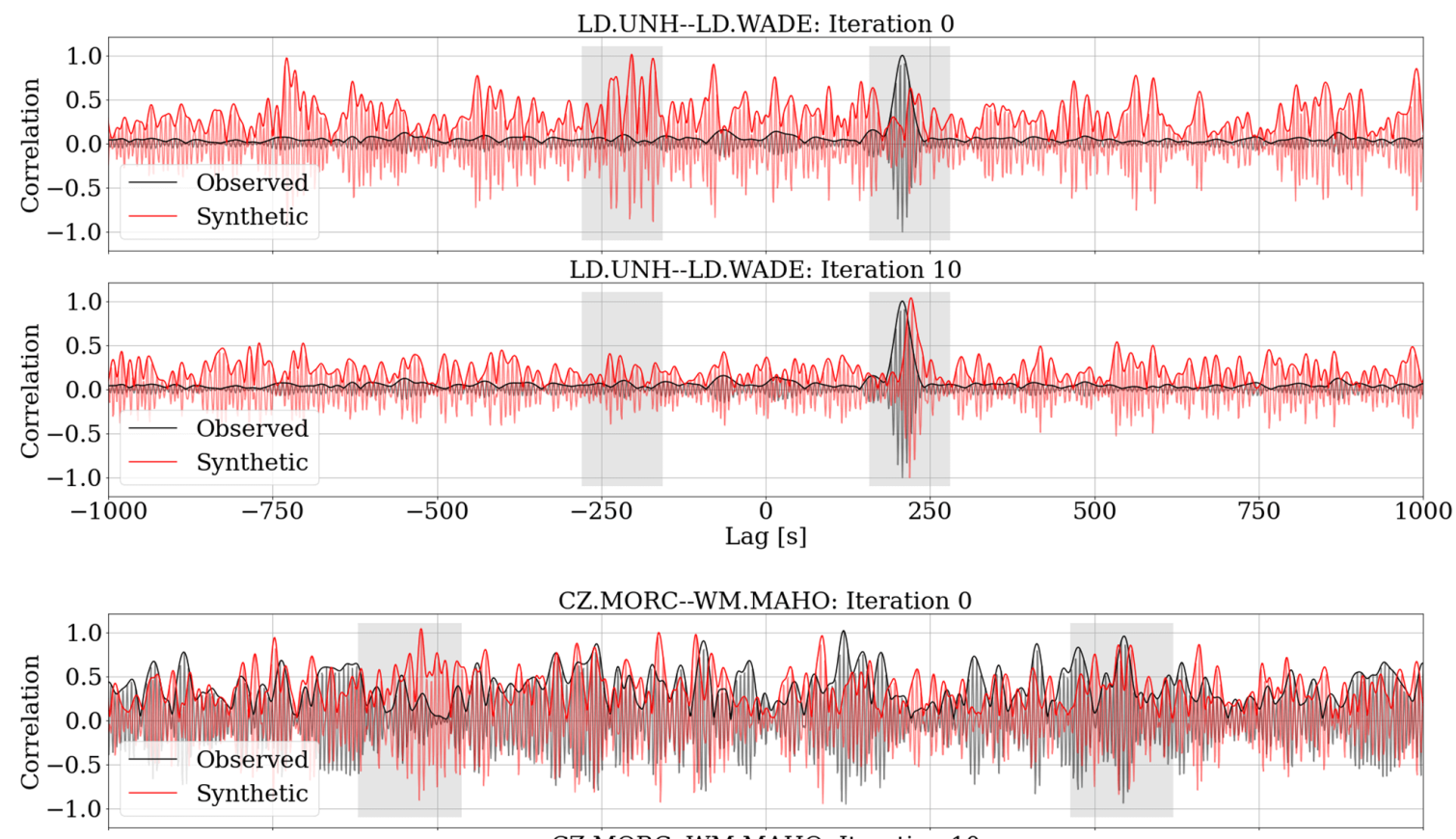

CZ.MORC--WM.MAHO: Iteration 10

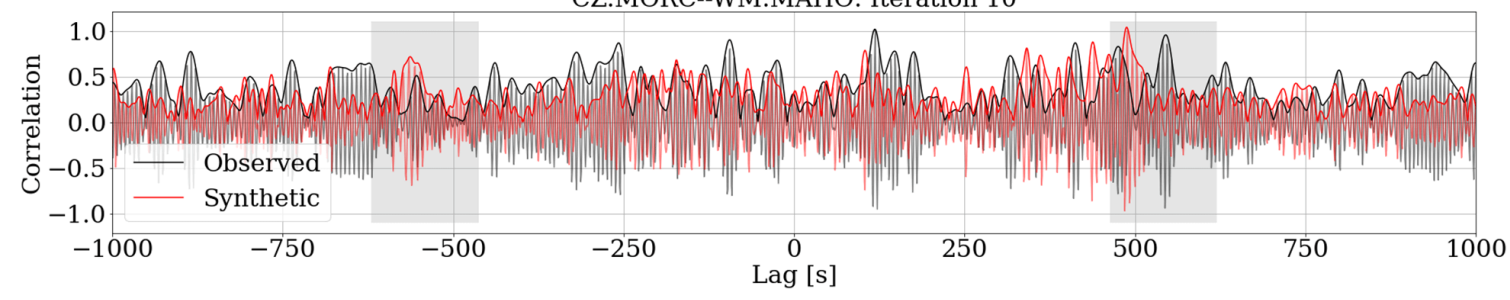

Figure 10. Observed and synthetic correlations for the homogeneous initial and the final PSD model. Measurement windows in the causal and acausal branches are indicated by the grey boxes. For better visibility, we also plot the correlation envelope. Correlation waveforms consisting of a simple surface wave arrival within the measurement window are fitted well (top). In contrast, when correlation waveforms are more complex, only the causal-to-acausal energy ratio is matched (bottom). This behaviour is expected as we do not attempt to fit waveforms but only the less informative but more robust energy ratio.

motion. Taking measurements on the horizontal components has been shown to improve results (Xu et al. 2019) and should be implemented in the future. Improving the measurement and using more information from the correlations is part of current research.

\subsection{3-D Earth structure, oceans and effective noise sources}

While the proposed method is in principle independent of the Earth model used to compute Green's functions, we work with radially symmetric Earth models in order to reduce computational cost to a feasible level. Even though it has been shown that the measurement of logarithmic energy ratios is 
robust against unknown or diregarded 3-D Earth structure (Sager et al. 2018a), this simplification may still affect the inversion results.

Along similar lines, and for the same reasons, we do not account for the presence of the oceans. The numerical modelling of global wave propagation with fluid ocean layers in the microseismic frequency range is currently out of scale. Future work may build on recent advancements that facilitate modelling with a fluid ocean layer and 3-D variable structure (Leng et al. 2019; Fernando et al. 2020). Alternatively, and most comprehensively, Sager et al. (2020) invert for both 3-D Earth structure and noise distribution simultaneously. This does, however, greatly increase the computational cost, particularly when considering high-frequency noise like the secondary microseisms.

In the same context, it is important to keep in mind that all inversion results - not only for the specific inverse problem considered here - a relative to generally unavoidable simplifications of forward modelling physics. In this sense, the source distributions that we obtain are an effective physical quantity. A well-known and related example are earthquake moment tensors, which are effective pointlocalised simplifications of a more complicated finite-rupture process, and which are mostly estimated on the basis of 1-D Earth models without fluid ocean layers. Despite being effective, the resulting sources are useful, e.g., for tectonic studies.

\subsection{Temporal averaging and temporal resolution}

Since the estimation of the source distribution is based on temporal averaging, we have to ensure that the length of the finite-time cross-correlations is sufficient to neglect stochastic fluctuations such as instrumental noise. Though we did not investigate the lower limit of temporal resolution, the low levels of instrumental noise in modern broadband seismometers, ensure that averaging over time scales that exceed the longest period by an order of magnitude, eliminates instrumental noise for all practical purposes.

The actual limitation of temporal resolution comes from the measurement process and our intuition for the inverse problem. These rest on the assumption that approximations of surface waves appear within the measurement windows. Hence, the averaging should be sufficiently long to ensure that surface waves become visible. Though this is technically not required, it still allows us to interpret the measurements and to set up the inverse problem accordingly. In our specific application, averaging over one day typically produces surface wave approximations that can be used in the logarithmic energy measurements. 


\subsection{Coverage and station locations}

The station coverage is of great importance for ambient noise studies as this determines the area of sensitivity. If we include continental grid points (see Fig. S3) we see inversion artifacts on the continents due to the lack of station coverage (and thus sensitivity) where we do not expect any sources. Choosing the station locations based on a more elaborate scheme using optimal design could further improve the resolution of the method whilst possibly decreasing the computational cost.

\subsection{Computational requirements}

For a large number of stations and global inversions, access to high performance computing facilities is useful as it significantly decreases the compute time due to the embarrassingly parallel nature of the code. A wavefield conversion with an AxiSEM wavefield including all frequencies up to $0.2 \mathrm{~Hz}$ for 150 stations and about 13,000 grid points takes between 1 and 4 hours per station depending on the sampling rate and correlation length. If we keep the station locations and the grid the same, this computation only has to be done once. A consequent inversion with 10 iterations using a subset of 3,100 cross-correlations from the 150 stations takes about 50 minutes on 600 cores. Smaller inversions on a local scale can easily be run on small clusters using publicly available pre-computed AxiSEM wavefields from, e.g., the Syngine repository (IRIS 2015; Krischer et al, 2017).

\section{CONCLUSIONS AND OUTLOOK}

We efficiently invert for the seismic noise distribution of the secondary microseisms by computing finite-frequency sensitivity kernels using a logarithmic energy ratio measurement of noise crosscorrelations. The computational cost is reduced by implementing a spatially variable grid to optimise the parameterisation, and by pre-computed wavefields from which Green's functions for forward modelling and kernel computations can easily be extracted. Benchmark tests show that these simplifications have no effect on the measurement we use. Several advantages arise from our approach: (i) we properly account for global wave propagation with visco-elastic attenuation; (ii) no assumptions on the wavefield (e.g. equipartioning or plane waves) have to be made; (iii) our measurement is robust to unknown 3D Earth structure; (iv) the method can be applied to any noise cross-correlation data set; and is (v) computationally inexpensive. Further possibilities for the spatially variable grid could include automatic grid adaptation based on either the chosen station locations, an improved starting model from other noise imaging methods like Matched-Field Processing (Gal et al. 2018; Bowden et al. 2020), or adapting the grid in subsequent iterations of the inversion.

Synthetic inversions show promising results and we are able to resolve the dominant noise sources 
of the target model. The spatial resolution is limited to a few hundred kilometres due to the spreading of the sensitivity kernel as we move away from the station pairs. Data inversions for several consecutive days demonstrate that we are able to see the spatio-temporal variations in the sources of secondary microseisms with our method. Further analysis should be done on more elaborate window picking and choosing the station locations based on an optimal design scheme. A synthetic inversion with globally distributed station locations (see Fig. S4) additionally demonstrates the potential for efficient noise source inversions with sensitivity all around the globe.

Due to the efficiency of the method, we pave the way for global daily ambient noise source inversions. Publicly available daily noise source maps could help to improve methods in full waveform ambient noise tomography and near real-time subsurface monitoring.

\section{ACKNOWLEDGMENTS}

The authors would like to thank Fabrice Ardhuin for providing the ocean surface pressure maps and Lucia Gualtieri for helping to implement the ocean site effect. Thanks to Korbinian Sager for providing the cross-correlations for the Salvus benchmark. LE gratefully acknowledges support from the Swiss National Science Foundation under grant P2EZP2_175124. Computations were run on Piz Daint at the Swiss National Supercomputing Centre (projects s741, s868, s961, and \$1040), and the help of their support team is much appreciated. Thanks to Krystyna Smolinski for the constant moral support and correcting grammar mistakes.

\section{Data and Code Availability}

The code and data are available from the authors upon request.

\section{REFERENCES}

Afanasiev, M., Boehm, C., van Driel, M., Krischer, L., Rietmann, M., May, D. A., Knepley, M. G., \& Fichtner, A., 2019. Modular and flexible spectral-element waveform modelling in two and three dimensions, Geophys. J. Int., 216(3), 1675-1692.

Aki, K., 1957. Space and time spectra of stationary stochastic waves, with special reference to microtremors, Bull.Earthq. Res. Inst., 35, 415-456.

Ardhuin, F. \& Herbers, T. H., 2013. Noise generation in the solid Earth, oceans and atmosphere, from nonlinear interacting surface gravity waves in finite depth, J. Fluid Mech., 716, 316-348.

Ardhuin, F., Stutzmann, E., Schimmel, M., \& Mangeney, A., 2011. Ocean wave sources of seismic noise, $J$. Geophys. Res. Ocean., 116(9), 1-21. 
Ardhuin, F., Gualtieri, L., \& Stutzmann, E., 2015. How ocean waves rock the Earth: Two mechanisms explain microseisms with periods 3 to 300s, Geophys. Res. Lett., 42(3), 765-772.

Ardhuin, F., Gualtieri, L., \& Stutzmann, E., 2019. Physics of ambient noise generation by ocean waves, in Seismic Ambient Noise, pp. 109-143, Cambridge University Press, Cambridge, U.K.

Bowden, D., Sager, K., Fichtner, A., \& Chmiel, M., 2020. Connecting Beamforming and Kernel-based Source Inversion, Geophys. J. Int., pp. 1-14.

Datta, A., Hanasoge, S., \& Goudswaard, J., 2019. Finite-Frequency Inversion of Cross-Correlation Amplitudes for Ambient Noise Source Directivity Estimation, J. Geophys. Res. Solid Earth, 124(7), 6653-6665.

Dziewonski, A. M. \& Anderson, D. L., 1981. Preliminary reference Earth model, Phys. earth Planet. Inter., 25(4), 297-356.

Ermert, L., Villasenor, A., \& Fichtner, A., 2016. Cross-correlation imaging of ambient noise sources, Geophys. J. Int., 204, 347-364.

Ermert, L., Sager, K., Afanasiev, M., Boehm, C., \& Fichtner, A., 2017. Ambient Seismic Source Inversion in a Heterogeneous Earth: Theory and Application to the Earth's Hum, J. Geophys. Res. Solid Earth, 122(11), 9184-9207.

Ermert, L., Igel, J., Sager, K., Stutzmann, E., Nissen-meyer, T., \& Fichtner, A., 2020. noisi : A Python tool for ambient noise cross-correlation modeling and noise source inversion, Solid Earth, preprint.

Fernando, B., Leng, K., \& Nissen-Meyer, T., 2020. Oceanic high-frequency global séismic wave propagation with realistic bathymetry, Geophys. J. Int., 222(2), 1178-1194.

Fichtner, A., 2014. Source and processing effects on noise correlations, Geophys. J. Int., 197(3), 1527-1531.

Fichtner, A. \& Tsai, V., 2019. Theoretical foundations of noise interferometry, in Seismic Ambient Noise, pp. 109-143, Cambridge University Press, Cambridge, U.K

Fichtner, A., Stehly, L., Ermert, L., \& Boehm, C., 2017. Generalized interferometry - I: Theory for interstation correlations, Geophys. J. Int., 208(2), 603-638.

Fichtner, A., Bowden, D., \& Ermert, L., 2020. Optimal processing for seismic noise correlations, Geophys. J. Int., p. in press.

Froment, B., Campillo, M., Roux, P., Gouédard, P., Verdel, A., \& Weaver, R. L., 2010. Estimation of the effect of nonisotropically distributed energy on the apparent arrival time in correlations, Geophysics, $\mathbf{7 5}$, SA85-SA93.

Gal, M., Reading, A. M., Rawlinson, N., \& Schulte-Pelkum, V., 2018. Matched Field Processing of ThreeComponent Seismic Array Data Applied to Rayleigh and Love Microseisms, J. Geophys. Res. Solid Earth, 123(8), 6871-6889.

Gerstoft, P. \& Tanimoto, T., 2007. A year of microseisms in southern California, Geophys. Res. Lett., 34(20).

Gizon, L. \& Birch, A. C., 2002. TimeDistance Helioseismology: The Forward Problem for Random Distributed Sources, Astrophys. J., 571(2), 966-986.

Gualtieri, L., Stutzmann, E., Capdeville, Y., Ardhuin, F., Schimmel, M., Mangeney, A., \& Morelli, A., 2013.

Modelling secondary microseismic noise by normal mode summation, Geophys. J. Int., 193(3), 1732-1745. 
Gualtieri, L., Stutzmann, E., Farra, V., Capdeville, Y., Schimmel, M., Ardhuin, F., \& Morelli, A., 2014. Modelling the ocean site effect on seismic noise body waves, Geophys. J. Int., 197(2), 1096-1106.

Halliday, D. \& Curtis, A., 2008. Seismic interferometry, surface waves and source distribution, Geophys. J. Int., 175, 1067-1087.

Hanasoge, S. M., 2013a. The influence of noise sources on cross-correlation amplitudes, Geophys. J. Int., 192(1), 295-309.

Hanasoge, S. M., 2013b. Measurements and kernels for source-structure inversions in noise tomography, Geophys. J. Int., 196(2), 971-985.

Hasselmann, K., 1963. A statistical analysis of the generation of microseisms, Rev. Geophys., 1(2), 177-210. Hillers, G., Graham, N., Campillo, M., Kedar, S., Landés, M., \& Shapiro, N., 2012. Global oceanic microseism sources as seen by seismic arrays and predicted by wave action models, Geochemistry, Geophys. Geosystems, 13(1).

Igel, H. \& Weber, M., 1996. P-SV wave propagation in the Earth's mantle using finite-differences: application to heterogeneous lowermost mantle structure, Geophys. Res. Lett., 23, 731-734.

IRIS, 2015. The iris synthetics engine.

Juretzek, C. \& Hadziioannou, C., 2016. Where do ocean microseisms come from? A study of Love-to-Rayleigh wave ratios, J. Geophys. Res. Solid Earth, 121(9), 1-16.

Kimman, W. P. \& Trampert, J., 2010. Approximations in seismic interferometry and their effects on surface waves, Geophys. J. Int., 182(1), 461-476.

Komatitsch, D. \& Tromp, J., 2002. Spectral-element simulations of global seismic wave propagation: I. Validation, Geophys. J. Int., 149(2), 390-412.

Krischer, L., Megies, T., Barsch, R., Beyreuther, M., Lecocq, T., Caudron, C., \& Wassermann, J., 2015. ObsPy: A bridge for seismology into the scientific Python ecosystem, Comput. Sci. Discov., 8(1), 0-17.

Krischer, L., Hutko, A. R., Van Driel, M., Stähler, S., Bahavar, M., Trabant, C., \& Nissen-Meyer, T., 2017. On-demand custom broadband synthetic seismograms, Seismol. Res. Lett., 88(4), 1127-1140.

Leng, K., Nissen-Meyer, T., van Driel, M. Hosseini, K., \& Al-Attar, D., 2019. AxiSEM3D: Broad-band seismic wavefields in 3-D global earth models with undulating discontinuities, Geophys. J. Int., 217(3), 2125 2146.

Lobkis, O. I. \& Weaver, R. L., 2001. On the emergence of the Green's function in the correlations of a diffuse field, J. Acoust. Soc, Am., 110(6), 3011-3017.

Longuet-Higgins, M. S., 1950. A Theory of the Origin of Microseisms, Philos. Trans. R. Soc. London, $\mathbf{2 4 3}(857), 1 \angle 35$.

Nakata, N., Gualtieri, L., \& Fichtner, A., 2019. Seismic Ambient Noise, Cambridge University Press.

Nishida, K. \& Fukao, Y., 2007. Source distribution of Earth's background free oscillations, J. Geophys. Res. Solid Earth, 112(B6).

Nissen-Meyer, T., van Driel, M., Stähler, S., Hosseini, K., Hempel, S., Auer, L., Colombi, A., \& Fournier, A., 2014. AxiSEM: broadband 3-D seismic wavefields in axisymmetric media, Solid Earth, (1), 425-445. 
Retailleau, L. \& Gualtieri, L., 2019. Toward High-Resolution Period-Dependent Seismic Monitoring of Tropical Cyclones, Geophys. Res. Lett., 46(3), 1329-1337.

Retailleau, L., Boué, P., Stehly, L., \& Campillo, M., 2017. Locating Microseism Sources Using Spurious Arrivals in Intercontinental Noise Correlations, J. Geophys. Res. Solid Earth, 122(10), 8107-8120.

Sabra, K. G., Gerstoft, P., Roux, P., Kuperman, W. A., \& Fehler, M. C., 2005. Surface wave tomography from microseisms in Southern California, Geophys. Res. Lett., 32(14), 1-4.

Sager, K., Boehm, C., Ermert, L., Krischer, L., \& Fichtner, A., 2018a. Sensitivity of Seismic Noise Correlation Functions to Global Noise Sources, J. Geophys. Res. Solid Earth, 123(8), 6911-6921.

Sager, K., Ermert, L., Boehm, C., \& Fichtner, A., 2018b. Towards full waveform ambient noise inversion, Geophys. J. Int., 212(1), 566-590.

Sager, K., Boehm, C., Ermert, L., Krischer, L., \& Fichtner, A., 2020. GlobalScale FullWaveform Ambient Noise Inversion, J. Geophys. Res. Solid Earth.

Sánchez-Sesma, F. J. \& Campillo, M., 2006. Retrieval of the Green's function from cross correlation; The canonical elastic problem, Bull. Seismol. Soc. Am., 96(3), 1182-1191.

Shapiro, N. M. \& Campillo, M., 2004. Emergence of broadband Rayleigh waves from correlations of the ambient seismic noise, Geophys. Res. Lett., 31, doi:10.1029/2004GL019491.

Shapiro, N. M., Campillo, M., Stehly, L., \& Ritzwoller, M. H., 2005. High-resolution surface-wave tomography from ambient seismic noise, Science (80-. )., 307(5715), 1615-1618,

Sladen, A., Rivet, D., Ampuero, J. P., De Barros, L., Hello, Y., Calbris, G., \& Lamare, P., 2019. Distributed sensing of earthquakes and ocean-solid Earth interactions on seafloor telecom cables, Nat. Commun., 10(1), $1-8$.

Stehly, L., Campillo, M., Froment, B., \& Weaver, R. L., 2008. Reconstructing Green's function by correlation of the coda of the correlation (C3) of ambient seismic noise, J. Geophys. Res. Solid Earth, 113(11), 1-10.

Tolman, H. L. \& Chalikov, D., 1996. Source terms in a third-generation wind wave model.

Tromp, J., Luo, Y., Hanasoge, S., \& Peter, D., 2010. Noise cross-correlation sensitivity kernels, Geophys. J. Int., 183(2), 791-819.

Tsai, V. C., 2009. On establishing the accuracy of noise tomography traveltime measurements in a realistic medium, Geophys. J. Int., 178, 1555-1564.

van Driel, M., Krischer, L., Stähler, S., Hosseini, K., \& Nissen-Meyer, T., 2015. Instaseis: Instant global seismograms based on a broadband waveform database, Solid Earth, 6(2), 701-717.

Virtanen, P., Gommers, R., Oliphant, T. E., Haberland, M., Reddy, T., Cournapeau, D., Burovski, E., Peterson, P., Weckesser, W., Bright, J., van der Walt, S. J., Brett, M., Wilson, J., Millman, K. J., Mayorov, N., Nelson, A. R., Jones, E., Kern, R., Larson, E., Carey, C. J., Polat, 1., Feng, Y., Moore, E. W., VanderPlas, J., Laxalde, D. Perktold, J., Cimrman, R., Henriksen, I., Quintero, E. A., Harris, C. R., Archibald, A. M., Ribeiro, A. H., Pedregosa, F., van Mulbregt, P., Vijaykumar, A., Bardelli, A. P., Rothberg, A., Hilboll, A., Kloeckner, A., Scopatz, A., Lee, A., Rokem, A., Woods, C. N., Fulton, C., Masson, C., Häggström, C., Fitzgerald, C., Nicholson, D. A., Hagen, D. R., Pasechnik, D. V., Olivetti, E., Martin, E., Wieser, E., Silva, F., Lenders, F., 
Wilhelm, F., Young, G., Price, G. A., Ingold, G. L., Allen, G. E., Lee, G. R., Audren, H., Probst, I., Dietrich, J. P., Silterra, J., Webber, J. T., Slavič, J., Nothman, J., Buchner, J., Kulick, J., Schönberger, J. L., de Miranda Cardoso, J. V., Reimer, J., Harrington, J., Rodríguez, J. L. C., Nunez-Iglesias, J., Kuczynski, J., Tritz, K., Thoma, M., Newville, M., Kümmerer, M., Bolingbroke, M., Tartre, M., Pak, M., Smith, N. J., Nowaczyk, N., Shebanov, N., Pavlyk, O., Brodtkorb, P. A., Lee, P., McGibbon, R. T., Feldbauer, R., Lewis, S., Tygier, S., Sievert, S., Vigna, S., Peterson, S., More, S., Pudlik, T., Oshima, T., Pingel, T. J., Robitaille, T. P., Spura, T., Jones, T. R., Cera, T., Leslie, T., Zito, T., Krauss, T., Upadhyay, U., Halchenko, Y. O., \& Vázquez-Baeza, Y., 2020. SciPy 1.0: fundamental algorithms for scientific computing in Python, Nat. Methods, 17(3), 261-272. Wapenaar, K., 2004. Retrieving the elastodynamic Green's function of an arbitrary inhomogeneous medium by cross correlation, Phys. Rev. Lett., 93(25), 1-4.

Wapenaar, K. \& Fokkema, J., 2006. Green's function representations for seismic interferometry, Geophysics, 71(4), SI33-SI46.

Weaver, R. L., 2008. Ward identities and the retrieval of Green's functions in the correlations of a diffuse field,

Wave Motion, 45, 596-604.

Weaver, R. L. \& Lobkis, O. I., 2004. Diffuse fields in open systems and the emergence of Green's function, $J$. Acoust. Soc. Am., 116, 2731-2734.

Williams, E. F., Fernández-Ruiz, M. R., Magalhaes, R., Vanthillo, R., Zhan, Z., González-Herráez, M., \& Martins, H. F., 2019. Distributed sensing of microseisms and teleseisms with submarine dark fibers, Nat. Commun., 10(1), 1-11.

Woodard, M. F., 1997. Implications of Localized, Acoustic Absorption for Heliotomographic Analysis of Sunspots, Astrophys. J., 485(2), 890-894.

Xu, Z., Dylan Mikesell, T., Gribler, G., \& Mordret, A., 2019. Rayleigh-wave multicomponent crosscorrelation-based source strength distribution inversion. Part 1: Theory and numerical examples, Geophys. J. Int., 218(3), 1761-1780.

Xu, Z., Dylan Mikesell, T., Umlauft, J., \& Gribler, G., 2020. Rayleigh-wave multicomponent crosscorrelationbased source strength distribution inversions. Part 2: A workflow for field seismic data, Geophys. J. Int. . 